\title{
Bewegung schützt vor kognitivem Abbau
}

Fragestellung: Kann regelmäßige körperliche Betätigung bei älteren Menschen die Entwicklung, das Auftreten und das Fortschreiten kognitiver Störungen verlangsamen?

Hintergrund: Vor dem Hintergrund einer zunehmend alternden Bevölkerung wird eines der größten Gesundheitsprobleme der Zukunft - nach malignen und kardiovaskulären Erkrankungen - die steigende Anzahl demenzieller Erkrankungen sein. Es ist in der Zwischenzeit unstrittig, dass vaskuläre Risikofaktoren nicht nur für die vaskuläre Demenz, sondern auch für degenerative Demenzerkrankungen wie die AlzheimerKrankheit eine wichtige Rolle spielen. Eine Reihe von Studien hat bereits einen Zusammenhang zwischen körperlicher Aktivität und kognitiven Störungen bei älteren Menschen gefunden. Es gibt allerdings wenige Studien, die den Einfluss der körperlichen Aktivität über einen längeren Zeitraum mit neuropsychologischen Tests und kernspintomografischen Untersuchungen analysiert haben. Die Northern Manhattan Studie (NOMAS) ist einzigartig, da sie viele Ethnien einschließt sowie bei 2.636 der 3.398 Studienteilnehmer zu Beginn auch eine Kernspintomografie und bei 1.228 Patienten eine neuropsychologische Testung durchgeführt worden war.

Patienten und Methodik: Die NOMAS ist eine populationsbezogene prospektive Kohortenstudie bei Menschen im Alter über 40 Jahren in Northern Manhattan, die zu dem Zeit-

Willey JZ, Gardener H, Caunca MR et al. Leisure-time physical activity associates with cognitive decline: The Northern Manhattan Study. Neurology 2016; 86: $1897-903$ punkt des Einschlusses in die Kohorte keinen Schlaganfall erlitten hatten. Bei 1.228 Teilnehmern wurde eine detaillierte neuropsychologische Untersuchung durchgeführt. Bei 876 Teilnehmern wurde fünf Jahre später eine zweite neuropsychologische Testung durchgeführt. Diese Untersuchung umfasste die Domänen Verarbeitungsgeschwindigkeit, semantisches Gedächtnis, episodisches Gedächtnis und exekutive Funktionen. Außerdem wurde die körperliche Aktivität in keine oder geringe versus regelmäßige und ausgeprägte körperliche Aktivität eingeteilt. Die letzte Gruppe machte $10 \%$ der Studienteilnehmer aus. Alle vorgestellten Analysen wurden für soziodemografische sowie kardiovaskuläre Risikofaktoren adjustiert.

Ergebnisse: Die Teilnehmer waren zum Zeitpunkt der kernspintomografischen Untersuchung im Mittel 70 Jahre alt und hatten eine im Mittel zehnjährige Schulbildung. Von den Studienteilnehmern wiesen 60-70\% eine arterielle Hypertonie und $15-20 \%$ einen Diabetes mellitus auf.

Zum Zeitpunkt der ersten neuropsychologischen Untersuchung fand sich ein eindeutiger Zusammenhang zwischen geringer körperlicher Aktivität und schlechteren Ergebnissen in den Bereichen Exekutivfunktionen, semantisches Gedächtnis und Verarbeitungsgeschwindigkeit. Von den Studienteilnehmern, die in der Baseline-Untersuchung kognitiv unauffällig waren, zeigen diejenigen, die sich wenig bewegten, über die folgenden fünf Jahre hinweg eine ausgeprägtere Verschlechterung der kognitiven Funktionen als Studienteilnehmer, die sich regelmäßig bewegten. Diese Effekte waren auch dann nachweisbar, wenn für soziodemografische Parameter und vaskuläre Risikofaktoren korrigiert wurde.

Schlussfolgerungen: Menschen mit geringer körperlicher Bewegung und Tätigkeit haben im Alter ein größeres Risiko, kognitive Funktionen einzubüßen, als Personen die sich regelmäßig bewegen.

\section{- Kommentar von Hans-Christoph Diener, Essen}

\section{Körperliche Aktivität in Therapieprogramme integrieren}

Die pharmazeutische Industrie hat in den letzten zehn Jahren Milliardenbeträge in große randomisierte Studien zur Prophylaxe und Therapie von Demenzen, insbesondere der Alzheimer-Demenz investiert. Diese Studien waren alle negativ. Die hier vorliegende prospektive Kohortenstudie zeigt, dass ganz offenbar eine regelmäßige körperliche Betätigung im höheren Lebensalter das Eintreten kognitiver Störungen verhindert und die Progression im zunehmenden Alter verlangsamt. Daher wäre es von extremer Wichtigkeit für ein Gesundheitssystem, alle Faktoren, die einer Demenz vorbeugen oder sie verlangsamen können, in Therapieprogramme auf der Ebene von Hausärzten, Internisten, Neurologen und Psychiatern zu integrieren. Dazu gehörten unter anderem eine aggressive Behandlung von vaskulären Risikofaktoren, eine regelmäßige geistige Betätigung, kognitives Training und auch regelmäßi- ge körperliche Aktivität. Diese muss natürlich an die gegebenen körperlichen Voraussetzungen älterer Menschen mit ihren diversen Begleiterkrankungen angepasst werden.

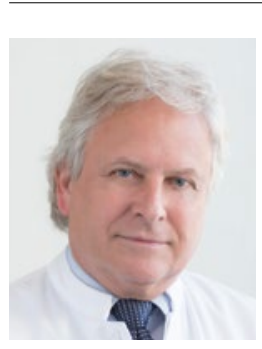

Prof. Dr. med. Hans-Christoph

Diener, Essen

Direktor der Klinik für Neurologie,

Universitätsklinikum Essen

E-Mail:h.diener@uni-essen.de 